\title{
Structural, Optical and Electrical Conductivity Properties of Stannite $\mathrm{Cu}_{2} \mathrm{ZnSnS}_{4}$
}

\author{
V.S. ZAKHVALINSKII, ${ }^{1,3}$ THI THAM HONG NGUYEN, ${ }^{1,4}$ \\ THI THAO PHAM ${ }^{1}$ NGOC TOAN DANG ${ }^{2,5}$ E.A. PILIUK, ${ }^{1}$ \\ and S.V. TARAN ${ }^{1}$ \\ 1.-Belgorod State National Research University, 85, Pobedy St., Belgorod, Russia 308015. \\ 2.-Institute of Research and Development, Duy Tan University, Da Nang 550000, Viet Nam. \\ 3.—e-mail: zakhvalinskii@bsu.edu.ru. 4.—e-mail: ngthamhong@gmail.com. 5.—e-mail: dangtoan \\ 2107@gmail.com
}

\begin{abstract}
A precursor powder was obtained from drying the solutions of a mixture of different ratios of $\mathrm{Cu}, \mathrm{Zn}$ and $\mathrm{Sn}$ chloride and thiourea. The $\mathrm{Cu}_{2} \mathrm{ZnSnS}_{4}$ (CZTS) samples were prepared from thermal decomposition of the corresponding precursors in vacuum, and were then characterized using scanning emission microscopy, energy dispersive x-ray analysis, x-ray powder diffraction and Raman scattering. According to x-ray diffraction analysis, all the synthesized samples had a tetragonal structure of space group $I \overline{4} 2 \mathrm{~m}$. The electrical properties of the CZTS samples were investigated in the temperature range of $10-325 \mathrm{~K}$. The charge carrier concentration was measured to be about $p=1 \times 10^{16} \mathrm{~cm}^{-3}$. A crossover from a nearest-neighbor hopping conduction mechanism at high temperatures $(T>150 \mathrm{~K})$ to a Mott variable-range hopping conduction mechanism at low temperatures $(T<150 \mathrm{~K})$ was observed. The activation energies of the CZTS samples were calculated for the low and high ranges of temperature. A thin film on quartz substrates was obtained using magnetron sputtering, for which a band gap of $E_{\mathrm{g}}=1.3 \mathrm{eV}$ was determined from transmittance measurements.
\end{abstract}

Key words: Chalcogenides, photovoltaic cells, high-absorption semiconductor, hopping conduction

\section{INTRODUCTION}

Our planet receives 10,000 times more solar energy than the total global energy consumption. ${ }^{1,2}$ Increasing solar energy processing is a very real problem. Reducing the cost of electricity generation is associated with the development of silicon technologies. Despite the great success and the positive dynamics of the development, the currently used materials and technologies have a number of drawbacks and limitations, which prevent the wide expansion of production. When using a photovoltaic cell layer of $\mathrm{CuIn}_{1-\mathrm{x}} \mathrm{Ga}_{\mathrm{x}}(\mathrm{S}, \mathrm{Se})_{2}$ (CIGS) and CdTe as a light-absorbing material, several points should be considered: $\mathrm{Cd}$ is a toxic metal, and Te, In and Ga are expensive due to their rarity (the earth's crust consists of

(Received September 12, 2016; accepted January 7, 2017; published online February 1, 2017)
$3 \times 10^{-5} \% \mathrm{Cd}, 10^{-3} \% \mathrm{Ga}, 10^{-5} \%$ In, $8 \times 10^{-5} \% \mathrm{Se}$ and $10^{-6} \%$ Te by weight). Consequently, the expansion of solar cell production based on these materials may be hopeless in terms of economics and ecology. To achieve a low-cost and environmentally friendly production of solar cells, some researchers have suggested using new materials not containing rare earth elements and toxic metals. It seems to be appropriate for environmental cleanliness and economic feasibility reasons to pay special attention to the substance $\mathrm{Cu}_{2} \mathrm{ZnSnS}_{4}$ (CZTS). ${ }^{3-7}$

CZTS is a promising material for applying in absorber layers of thin-film solar cells. Thin CZTS films have a suitable band gap of $1.4-1.5 \mathrm{eV}$ and a high optical absorption coefficient of $10^{4} \mathrm{~cm}^{-1}$. According to the Shockley-Queisser detailed balance limit and Standard Tables for References Solar Spectral ASTM G173 AM1.5GT at a temperature of $298 \mathrm{~K}$, the maximum achievable efficiency of a solar 


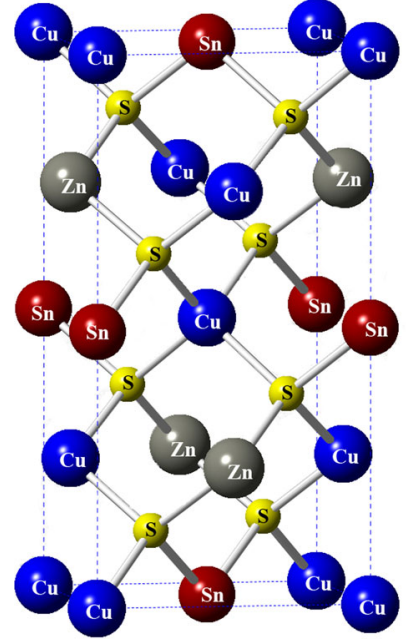

Kesterite

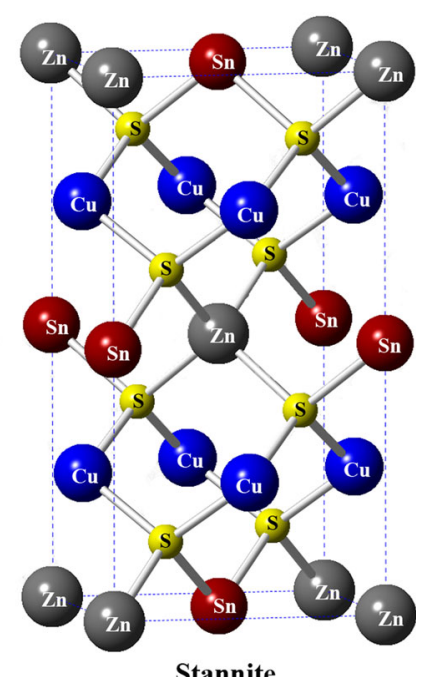

Stannite
Fig. 1. The conventional tetragonal unit cells of kesterite (space group $\overline{14}$ and stannite (space group $\overline{1} 2 \mathrm{~m}$ ).

cell using a $p-n$ junction was $\eta=32.2 \%^{3}$ and $32.4 \%^{4}$ for single-junction devices. These results can be considered promising, because the theoretical limit of efficiency of single-junction solar cells, also known as the limit of Shockley-Queisser, is $\eta=34 \% .8$

The kesterite (space group I $\overline{4}$ ) and stannite (space group $I \overline{4} 2 m$ ) are basic structures of CZTS (Fig. 1). The kesterite structure differs from the stannite in the location of the $\mathrm{Cu}$ and $\mathrm{Zn}$ cations that are more disordered, which leads to a lower symmetry of the structure. It has been found that the kesterite structure has the lowest energy. However, the difference in ground state energy between these structures is insignificant $(2-3 \mathrm{meV} / \mathrm{atom}){ }^{10}$ The calculated band diagrams for kesterite and stannite structures of CZTS show that the material has a direct energy band gap, ${ }^{11}$ which reaches $1.487 \mathrm{eV}$ for the kesterite structure and $1.295 \mathrm{eV}$ for the stannite structure. ${ }^{12}$ These values are close to optimal for the conversion of solar energy (from $1.0 \mathrm{eV}$ to $1.5 \mathrm{eV}$ ). ${ }^{13}$ Recently, experimental prototypes of photovoltaic structures based on CZTS with a record value of solar energy conversion efficiency of $9.2 \%$ were constructed. ${ }^{14}$

Although CZTS compounds have received increasing interest since the late 2000s for applications in solar cells, only a relatively few studies have reported the characteristics of CZTS with the stannite structure. Therefore, in this work, we synthesized different CZTS samples with the stannite structure and obtained thin films using magnetron sputtering. The structure, phase composition, optical properties and electrical conduction mechanisms of stannite CZTS were investigated.

\section{EXPERIMENTAL DETAILS}

\section{Synthesis of Bulk and Thin Films of CZTS}

There are various methods to synthesize monocrystalline CZTS, such as precipitation from solution, electro-precipitation, impulse laser precipitation and microwave-assisted precipitation. Thin film of CZTS can be obtained from spray pyrolysis, magnetron sputtering or different modifications of chemical vapor deposition (CVD). ${ }^{15}$ In this paper, the synthesis of bulk CSTZ was carried out using the method of pyrolytic decomposition of a stoichiometric mixture of chlorides and thiourea. Thin films of CZTS were obtained using the magnetron sputtering method.

The technology of the synthesis of the material is as follows: dissolving $\mathrm{CuCl}_{2} \cdot 2 \mathrm{H}_{2} \mathrm{O}, \mathrm{ZnCl}_{2}$, $\mathrm{SnCl}_{2} \cdot 2 \mathrm{H}_{2} \mathrm{O}$ and thiourea $\mathrm{SC}\left(\mathrm{NH}_{2}\right)_{2}$ in a $50 \%$ aqueous ethanol solution, then using an ultrasonic bath to obtain a homogeneous solution. CZTS precursors were obtained by drying the solution at $70-80^{\circ} \mathrm{C}$ for $48 \mathrm{~h}$. In order to achieve a greater dispersion, the dry powders were pulverized in a mortar. In the next step, the powders were annealed in a vacuum oven at $450^{\circ} \mathrm{C}$ for half an hour with constant removing of the pyrolytic decomposition byproducts. Three CZTS samples were obtained with the following atomic ratios of metals in the precursors: $[\mathrm{Zn}] /[\mathrm{Sn}]=1.25$ and $[\mathrm{Cu}] /([\mathrm{Zn}]+[\mathrm{Sn}])=1 ; 0.9$ and 1.1. The samples were designated as CZTS 1, CZTS 2 and CZTS 3, respectively.

The synthesized powders of CZTS 2 were used as the target for magnetron sputtering in producing thin films. Sample CZTS 2 was chosen because its metal atomic ratios are close to the optimal composition of solar cells. ${ }^{16}$ The powder was mixed with alcohol in a copper cuvette, then dried in an oven at $80^{\circ} \mathrm{C}$ for $30 \mathrm{~min}$. A sufficiently homogeneous and compact target was obtained.

The formation of a CZTS thin film can be divided into two stages. In the first stage, a thin film was deposited on quartz substrates using magnetron sputtering in an argon atmosphere at a pressure of $3.8 \times 10^{-3}$ mbar for $20 \mathrm{~min}$, with an average current of $10 \mathrm{~A}$ and a power of $100 \mathrm{~W}$. The distance between the target and the substrate was $80 \mathrm{~mm}$. The temperature of the substrate was kept at $350^{\circ} \mathrm{C}$. In the second stage, the film was sulfurized in a tubular furnace in an atmosphere of $\mathrm{N}_{2}$ and sulfur vapor at $400^{\circ} \mathrm{C}$ for $30 \mathrm{~min}$ to compensate for the loss of sulfur during the annealing process.

\section{Characterization Techniques}

The chemical composition of the obtained materials was studied using scanning electron microscopy (SEM). The phase composition and crystal structure of the CZTS samples were studied by x-ray diffraction (XRD) and Raman spectroscopy. XRD analysis of bulk samples was carried out on a Rigaku IV diffractometer equipped with a $\mathrm{D} /$ teX Ultra detector in the range of $10-100^{\circ}$, at a speed of $2 \% \mathrm{~min}$, $\lambda_{\mathrm{CuK}_{\alpha}}=1.54056 \AA$, without a monochromator, in Bragg-Brentano $(\theta-2 \theta)$ geometry. Raman spectra were studied using an XploRA ONE spectrometer with the wavelength of the solid excitation laser 
$\lambda=785 \mathrm{~nm}$. A study of the excitation and emission spectra of polycrystalline CZTS was also carried out. The band gap energy of the CZTS films was determined from transmittance measurements using an SF-2000 spectrophotometer in the range from $190 \mathrm{~nm}$ to $1000 \mathrm{~nm}$.

Studies on the temperature dependence of the resistivity $\rho(\mathrm{T})$ were carried out using the fourpoint probe method. Samples obtained by the above-mentioned techniques, pressing, grinding, cutting and polishing were given a rectangular shape with dimensions: $3.5 \times 9.8 \times 3.5 \mathrm{~mm}^{3}$ $($ CZTS 1$) ; 3.2 \times 9.9 \times 2.8 \mathrm{~mm}^{3}($ CZTS 2$) ; 2.9 \times$ $8.0 \times 2.7 \mathrm{~mm}^{3}$ (CZTS 3). Measurements were carried out at temperatures ranging from 10 to $325 \mathrm{~K}$ with a step of $2 \mathrm{~K}$. The contacts were created using silver paste. The experiment was carried out using a unit consisting of a Janis CCS-350C helium cryostat and a Lakeshore 331 temperature controller with an accuracy of within $\sim 1 \mathrm{~K}$. The values of the resistivity were recorded in the automatic mode of the program LabVIEW 7.0. The measurement of the Hall coefficient $R_{\mathrm{H}}$ was performed at room temperature in a constant magnetic field (1 T) with a constant current through the sample (7 mA).

\section{RESULTS AND DISCUSSION}

\section{Structural Study of the CZTS Samples}

Elemental analysis of the samples by using energy dispersive $\mathrm{x}$-ray spectroscopy (EDX) revealed that these compounds are non-stoichiometric, being sulfur-deficient due to the loss of sulfur during the high-temperature annealing process. XRD patterns of the compounds are shown in Fig. 2. Experimental data of the XRD experiments were analyzed by the Rietveld method using the FULLPROF program. ${ }^{17}$ The diffraction data refinement shows that the compounds crystallize in a tetragonal structure with symmetry $I \overline{4} 2 \mathrm{~m}$. The obtained structural parameters are given in Table I. The ratios $c / 2 a$ for all the samples CZTS 1,2 , and 3 are close to 1 , indicating the small degree of tetragonal distortion of these compounds.

Additional information about the phase composition of the samples was obtained by analyzing the Raman spectra. Figure 3 shows the Raman spectra for the samples CZTS 1, 2, 3 and the fitted curve of the Raman spectra of sample CZTS 1 using Gaussian functions.

The calculated vibrational modes for the Raman spectra of the typical kesterite structure of CZTS are $289 \mathrm{~cm}^{-1}, 339 \mathrm{~cm}^{-1}, 350 \mathrm{~cm}^{-1}$ and $370 \mathrm{~cm}^{-1}$ (modes of symmetry of type A). ${ }^{18}$ The Raman spectra obtained in our experiment (Fig. 3) have their most intense peaks located at $232 \mathrm{~cm}^{-1}$, $285 \mathrm{~cm}^{-1}$ and $334 \mathrm{~cm}^{-1}$. These peaks are assumed to associate with the modes of symmetry of type A1. According to the analysis in Ref. 19, these peaks

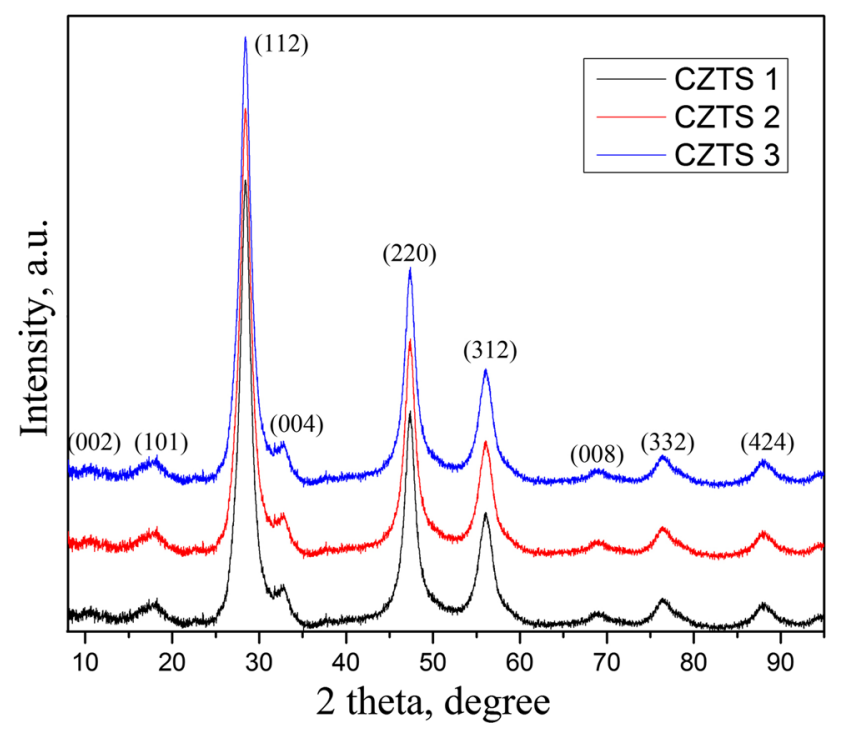

Fig. 2. Powder diffraction patterns of the samples CZTS 1, 2, 3.

Table I. Unit cell parameters and crystallite size of the CZTS samples

\begin{tabular}{|c|c|c|c|}
\hline \multirow[b]{2}{*}{ Sample } & \multicolumn{2}{|c|}{ Unit cell parameters } & \multirow[b]{2}{*}{ Ratio $c / 2 a$} \\
\hline & $a=b(\AA)$ & $c(\AA)$ & \\
\hline CZTS 1 & $5.431(2)$ & $10.928(6)$ & 1.006 \\
\hline CZTS 2 & $5.432(1)$ & $10.903(7)$ & 1.004 \\
\hline CZTS 3 & 5.435 (1) & $10.928(3)$ & 1.005 \\
\hline
\end{tabular}

are attributed to the tetragonal stannite-type structure with space group $I \overline{4} 2 \mathrm{~m}$. It should be noted that the Raman spectrum of the sample CZTS 3, which contains a slightly excessive amount of copper, differs from the other spectra in the reduced intensity of the second peak. The emission and excitation spectra of polycrystalline CZTS were also studied (Fig. 4). The shift of the fluorescence spectrum to the side of longer wavelengths compared to the absorption spectrum can be explained by non-radiative relaxation processes. As a result of these processes, part of the energy of the absorbed photon is lost, so the emitted photon has less energy. Figure 4 shows that the CZTS samples 1, 2 and 3 exhibit a marked fluorescence with a maximum at the wavelength of $\lambda=440 \mathrm{~nm}$. This maximal fluorescence emission approximately corresponds to the wavelength of blue light. In the synthesis technology used in this work, the presence of certain amounts of $\mathrm{ZnS}$ can be allowed, which is indicated by photoluminescence peak at the wavelength of $\lambda=440 \mathrm{~nm}$. This obtained value is in concordance with values found in the literature. $^{20}$ 

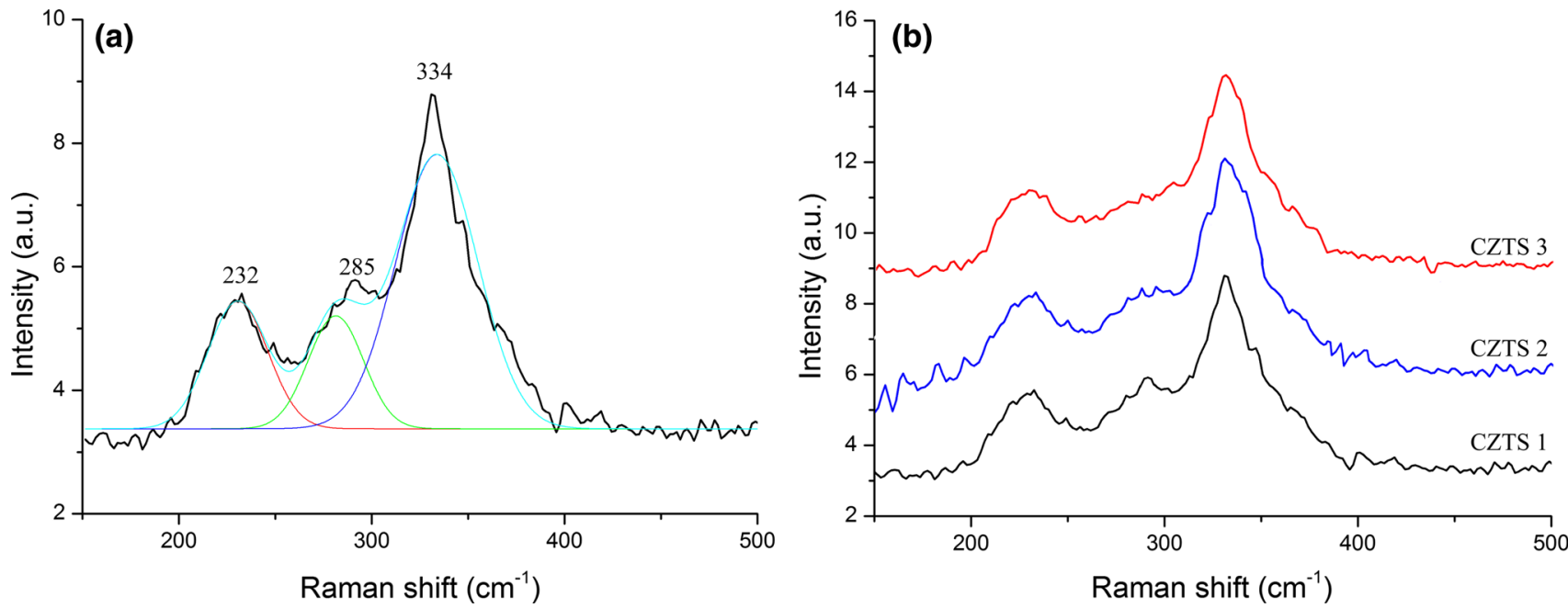

Fig. 3. (a) Raman spectra with peak fitting for sample CZTS 1 using Gaussian function (dashed line), (b) comparing the Raman spectra of samples CZTS 1, 2, 3.

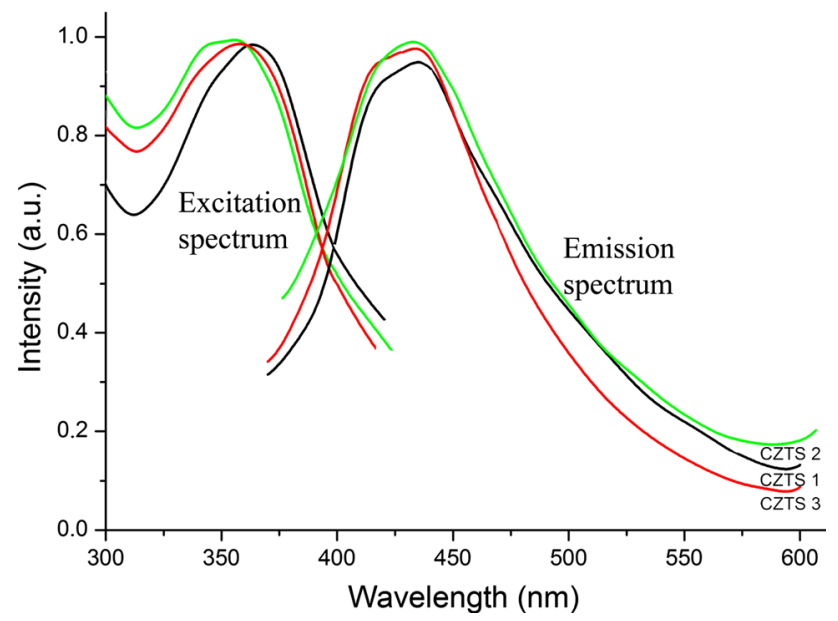

Fig. 4. Excitation and emission spectra of polycrystalline samples CZTS 1,2 and 3.

\section{Investigation of Electrical Conduction Mech- anisms of CZTS Samples}

The resistivity versus temperature data presented in Fig. 5 shows that the resistivity of the samples decreases with the increase of the atomic ratio of $[\mathrm{Cu}] /([\mathrm{Zn}]+[\mathrm{Sn}])$. In the investigated range of temperature, the activation nature of the conductivity was observed for all CZTS samples. It is possible to allocate two areas of conductivity, high temperature in the range of $150-325 \mathrm{~K}$ and low temperature in the range of $10-150 \mathrm{~K}$.

The analysis of the temperature dependence of the resistivity $\rho(T)$ at high temperatures was performed following the universal law ${ }^{21}$ :

$$
\rho(T)=\rho_{0} \exp \left(\frac{E_{\text {act }}}{k T}\right)
$$

where $\rho_{0}$ is a pre-exponential factor, $E_{\text {act }}$ is the conductivity activation energy and $k$ is the

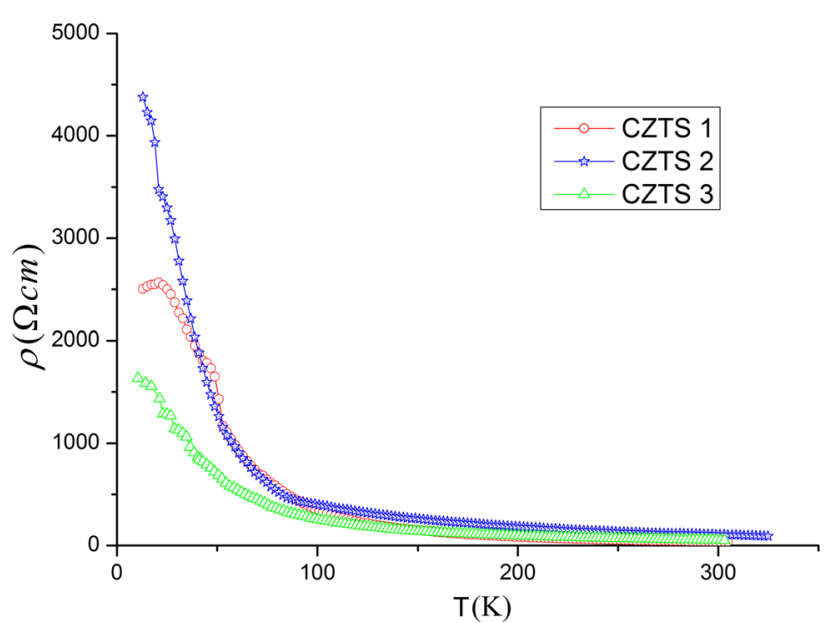

Fig. 5. Temperature dependence of the resistivity of samples CZTS 1,2 and 3.

Boltzmann constant. In the case of band conduction (activation of the main carriers from the level formed by defects to the conduction zone), $\rho_{0}$ does not depend on temperature. In the case of hopping conduction over the nearest neighbors, $\rho_{0}=A T$, where $A$ is a proportionality constant independent of temperature. Using Eq. 1, the conduction mechanism can be determined from the linearized plots of $\ln (\rho)$ versus $(1 / T)$ and $\ln (\rho / T)$ versus $(1 / T)$.

Analysis of the low-temperature area was carried out using a universal equation, which is often used in the case of crystalline semiconductors in the hopping conduction zone ${ }^{21}$ :

$$
\rho(T)=A_{\mathrm{p}} T^{1 / p} \exp \left[\left(\frac{T_{0 p}}{T}\right)^{1 / p}\right]
$$

where $A_{\mathrm{p}}$ is a constant pre-exponential factor, $T_{0 \mathrm{p}}$ is the characteristic temperature of hopping, $p=1$ corresponds to the conduction mechanism of 
hopping over nearest-neighbors (or the NNH conductivity), $p=4$ corresponds to the Mott variablerange hopping (VRH) mechanism and $p=2$ is associated with the Shklovskii-Efros VRH conduction mechanism. ${ }^{21}$ Let us point out that, for $p=1$, Eq. 2 coincides with Eq. 1. Lowering the temperature is energetically advantageous for the carriers making jumps with end points outside the closest centers, which leads to the variable-range hopping conduction mechanism. In this case, it is energetically more favorable for charge carriers to tunnel beyond the neighboring states.

The type of hopping conductivity was also determined by analysis of the local activation energy $E_{\text {loc }}$. The concept of local activation energy was first introduced by Shklovskii and Efros in their book The electronic properties of doped semiconductors. ${ }^{21}$ $E_{\text {loc }}$ is determined from the equation:

$$
E_{\mathrm{loc}}(T)=\frac{d[\ln \rho(T)]}{d(k T)^{-1}}
$$

Then:

$$
\ln \left(\frac{E_{\text {loc }}}{k T}+\frac{1}{p}\right)=\ln \left(\frac{1}{p}\right)+\frac{1}{p} \ln T_{0 \mathrm{p}}+\frac{1}{p} \ln \left(\frac{1}{T}\right)
$$

The left hand side of Eq. 4 represents a linear function of $\ln (1 / T)$ for a single value of $p$. Therefore, by putting the value of $p=1,2,4$ and from the slope of the linear section of the dependence $\ln \left(\frac{E_{\mathrm{loc}}}{k T}+\frac{1}{p}\right)$ versus $\ln (1 / T)$, the type of the hopping conductivity can be determined. Substitution of the values $p=1$, 2 and 4 into Eq. 4 allows determination of the temperature ranges, which correspond to different types of conductivity.

Furthermore, the value of $p$ was determined by minimizing the percentage of deviation (PD) by the equation: ${ }^{22}$

$$
\mathrm{PD}(\%)=\left\{\frac{1}{n} \sum_{i=1}^{n}\left[\frac{100}{\rho\left(T_{\mathrm{i}}\right)}\left\langle\rho_{0}\left(T_{i}\right) \exp \left(\frac{T_{0}}{T_{\mathrm{i}}}\right)^{s}-\rho\left(T_{\mathrm{i}}\right)\right\rangle\right]^{2}\right\}^{1 / 2}
$$

where $\rho\left(T_{i}\right)$ are experimental values of the resistivity at temperatures $T_{\mathrm{i}}$, and $n$ is the number of experimental data points. In the original method, it can be supposed that $\rho_{0}(T)=\rho_{0}=$ const. Then, the values $\rho_{0}$ and $T_{0}$ can be determined from the dependence $\ln (\rho)$ versus $T^{-s}$. Construction of the PD function at different values of $s$ (from 0.1 to 1 ) allows the determination of the value $p=1 / s$, which corresponds to the type of conductivity.

We first present the analysis of the resistivity data at high temperatures (150-325 K). If the thermodynamic energy of the system is estimated as $k T$ in this temperature range, it will take values from $13 \mathrm{meV}$ to $28 \mathrm{meV}$. The activation energies estimated from the linearization of $\ln (\rho)$ versus (1/ $T$ ) are too small compared to the thermodynamic energy of the system. Thus, we can assert, with a

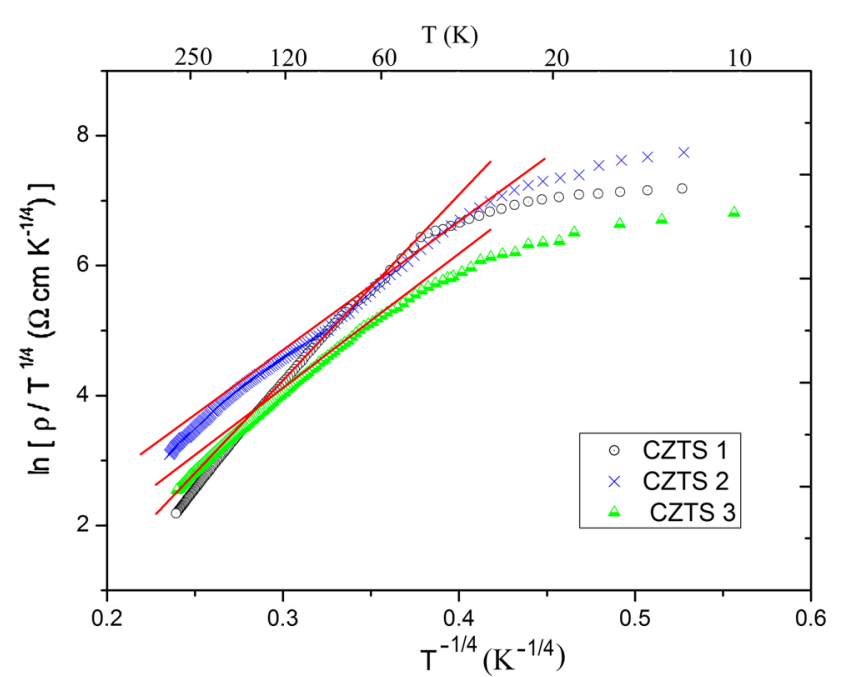

Fig. 6. $\ln \left(\rho / T^{1 / 4}\right)$ versus $T^{-1 / 4}$ for investigated samples CZTS 1, 2, 3. Solid lines linear fit of the experimental data.

high probability, the crossover from the nearestneighbor hopping conduction mechanism. The calculated activation energies for samples CZTS 1, 2 and 3 are $49 \mathrm{meV}, 42 \mathrm{meV}$, and $43 \mathrm{meV}$, respectively.

The hopping conductivity of the compound was further studied through the resistivity measurements at low temperatures (10-150 K). According to existing theoretical ideas, the CZTS conduction mechanism at low temperatures can be determined using the method of linearization $\rho(T)$ of Eq. 2. By putting the values of $p=1,2$ and 4 sequentially into the equation, the best linearity of the plots was obtained with $p=4$ within a much wider temperature interval (Fig. 6). This corresponds to the Mott VRH conductivity of all the investigated samples.

The linear part of the plots of $\ln \left(\rho / T^{1 / 4}\right)$ versus $T^{-1 / 4}$ in Fig. 6 yields the values of the characteristic temperature $T_{04}$ and $A_{4}$, as well as those of the onset temperature $T_{\mathrm{v}}$ (the upper limit of the Mott VRH conductivity regime), and the lowest temperature $T_{\mathrm{m}}$, which are listed in Table II for all three samples.

The important parameter $W_{0}$ shown in Table II, calculated using the formula:

$$
W_{0}=k\left(T_{\mathrm{v}}^{3} \cdot T_{04}\right)^{1 / 4},
$$

is associated with the width of the impurity band. Taking the bandwidth into consideration, we can estimate the value of the energy of the acceptor level: $W_{1} \approx 48 \mathrm{meV}, W_{1} \approx 42 \mathrm{meV}$, and $W_{1} \approx 41$ meV for samples CZTS 1, 2 and 3. For our samples, the ones with stannite structure, the energies of the acceptor level appear to be about double that of the kesterite structure of CZTS. ${ }^{23}$

Figure 7 shows the dependence of $\ln \left(\frac{E_{\text {loc }}}{k T}+\frac{1}{4}\right)$ versus $\ln (1 / T)$ for all three samples. 
Table II. The pre-exponential constant $A_{4}$, the onset temperature $T_{\mathrm{v}}$ and the lowest temperature $T_{\mathrm{m}}$ of the Mott VRH conductivity, the Mott VRH characteristic temperature $T_{04}$ and the bandwidth parameter $W_{0}$ for samples CZTS 1, 2, 3

\begin{tabular}{|c|c|c|c|c|c|}
\hline Sample & $T_{\mathbf{v}}(\mathbf{K})$ & $T_{\mathbf{m}}(\mathbf{K})$ & $A_{4}\left(\Omega \mathrm{cm} \mathrm{K}^{-1 / 4}\right)$ & $T_{04}^{1 / 4}\left(K^{1 / 4}\right)$ & $W_{0}(\mathrm{meV})$ \\
\hline CZTS 1 & 140 & 46 & 0.015 & 27.5 & 96 \\
\hline CZTS 2 & 180 & 50 & 0.246 & 19.9 & 84 \\
\hline CZTS 3 & 158 & 52 & 0.088 & 21.51 & 82 \\
\hline
\end{tabular}

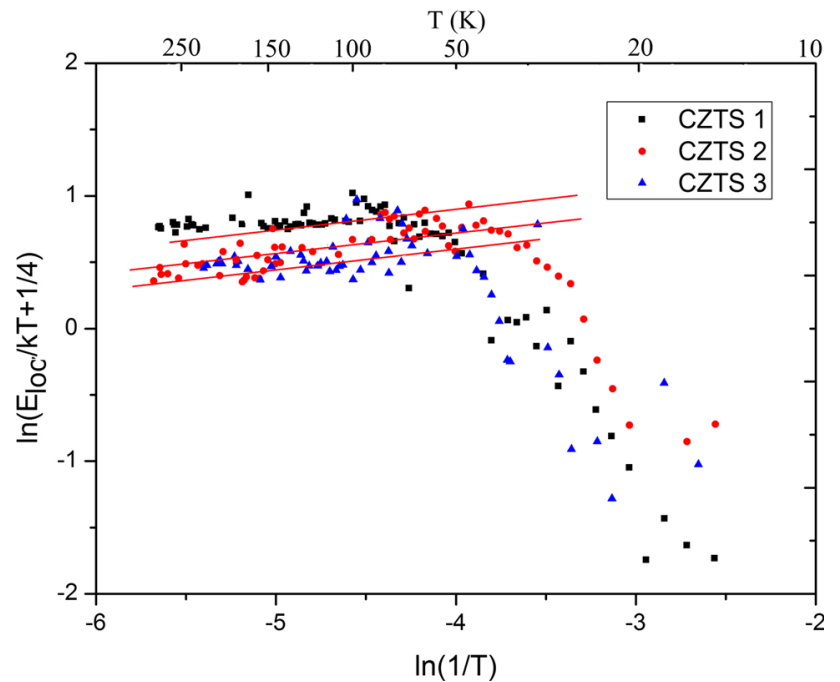

Fig. 7. $\ln \left(\frac{E_{l o c}}{k T}+\frac{1}{4}\right)$ versus $\ln (1 / T)$ for samples CZTS 1, 2, 3. Solid lines the linear fit with a fixed slope of $1 / 4$.

It can be seen from Fig. 7 that the data plot of $\ln \left(\frac{E_{\text {loc }}}{k T}+\frac{1}{4}\right)$ versus $\ln (1 / T)$ can be fitted to a linear relationship, giving $p=4$. The Mott VRH conduction mechanism can be deduced from the data using this method. In addition, such plots yield the temperature intervals $\left(T_{\mathrm{v}}, T_{\mathrm{m}}\right)$, which are close to the corresponding characteristic parameters in Table II.

Additionally, a correspondence of our resistivity data to the Mott regime of the VRH conduction was evaluated by minimizing the PD. The variation of $\mathrm{PD}$ as a function of the parameters $s=1 / p$ for all three samples is shown in Fig. 8, in which $s$ was varied from 0 to 1 . The best value of $s$ is the one which gives a minimum of the percentage deviation of the fitted curve to experimental points $\rho\left(T_{\mathrm{i}}\right)$. It is clear that the best fits are obtained for the value of $s$ around 0.25 . It means that, in order to determine the mechanism of conduction, the best fit is obtained using $p=4$ for all samples. This corresponds to the Mott VRH conductivity.

Thus, the types of conductivity and the characteristic parameters $T_{\mathrm{v}}, T_{\mathrm{m}}, A_{\mathrm{p}}, T_{0 \mathrm{p}}$, determined from three different methods, are similar. It was found that the behavior of the resistivity of the samples CZTS 1,2 , and 3 in the low-temperature range corresponds to the Mott mechanism of VRH conductivity.

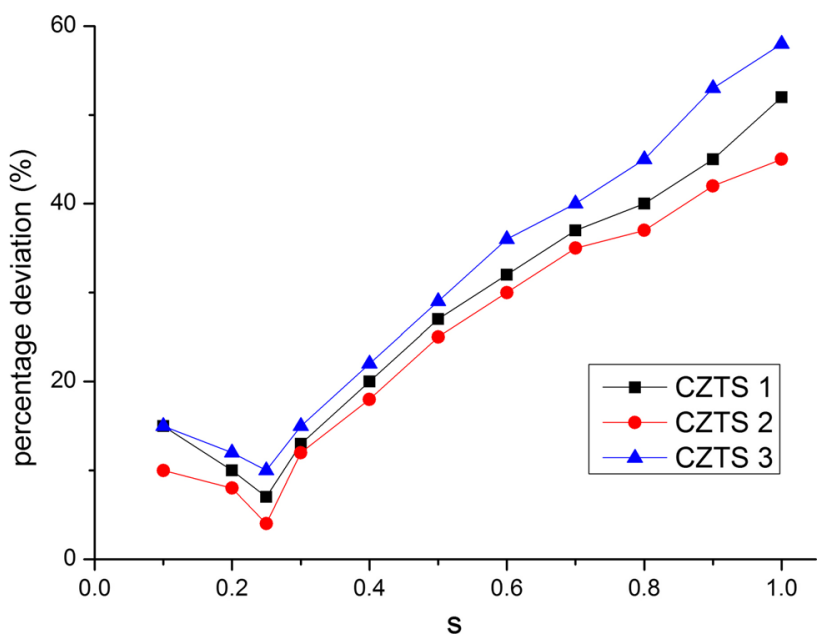

Fig. 8. The percentage deviation as a function of the parameter $s$ for the samples CZTS 1, 2, 3 .

Based on the measurement of the Hall coefficient, it was determined that the obtained semiconductor samples had $p$-type conductivity. The carrier concentration of the samples CZTS 1,2 and 3 is approximately $p \sim 10^{16} \mathrm{~cm}^{-3}$.

\section{Optical Study of Thin Films of CZTS}

The optical band gap of thin film is one of the key factors determining the efficiently of solar cells. The band gap was estimated based on measurements of the transmittance $\mathrm{T}$ in the range of wavelengths of $\lambda=190-1000 \mathrm{~nm}$ using the SF-2000 spectrophotometer.

Near the absorption edge or in the strong absorption zone of the transmittance spectra, there exists a relationship ${ }^{24}$ :

$$
(\alpha h v)^{1 / n}=A\left(h v-E_{\mathrm{g}}\right)
$$

where $\alpha$ is the absorption coefficient, $h v$ is the energy of the photon, $A$ is a constant, $E_{\mathrm{g}}$ is the optical band gap energy and $n$ is an index which characterizes the optical absorption process. $n$ can take a value of 2 or 1/2 for indirect-allowed or directallowed transitions, respectively. Since the probability of indirect transitions of photovoltaic is very small, we can choose $n$ as $1 / 2$. This means that the band gap can be estimated by extrapolating the linear part of the spectrum $(\alpha h v)^{2}=f(h v)$ to zero. 


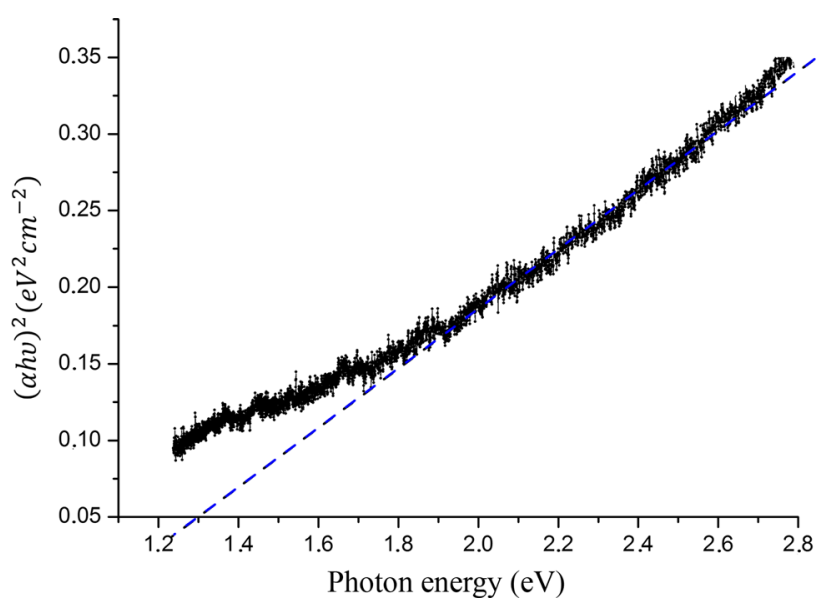

Fig. 9. $(\alpha h v)^{2}$ the versus photon energy $h v$ of CZTS film.

On the other hand, in electromagnetic theory the transmittance is calculated using the formula ${ }^{25}$ :

$$
T=(1-R)^{2} \cdot e^{-\alpha d}
$$

where $R$ is the reflectance and $d$ is the thickness of the film.

Hence, the absorption coefficient of the film is proportional to the logarithm of the transmittance: $\alpha \approx-\ln T$. Thus, the band gap of a CZTS film on a quartz substrate is determined by extrapolating the linear region of the plot $(-\ln T)^{2}$ versus the energy of photon $(h v)$ to the horizontal axis and taking the intersecting point.

In Fig. 9, we show the band gap estimation for a CZTS film. The band gap was found to be $E_{\mathrm{g}}=1.3 \mathrm{eV}$. This value is in good agreement with theoretical calculations. ${ }^{26}$ Furthermore, this energy is in the optimum range of band gaps for a solar cell. ${ }^{13}$ This optical property shows the prospect of CZTS for solar cells with high efficiency.

\section{CONCLUSIONS}

This article is devoted to our studies of the structure, phase composition, optical properties, and electrical conduction mechanisms of the semiconductor $\mathrm{Cu}_{2} \mathrm{ZnSnS}_{4}$. The synthesis of CZTS was performed using the method of pyrolytic decomposition of a stoichiometric mixture of the metal chlorides and thiourea. XRD patterns of the CZTS samples show that all of them have stannite structure which was confirmed by Raman analysis. The resistivity measurements of three CZTS samples depending on temperature in the range of 10-325 K were conducted. At high temperatures $(T>150 \mathrm{~K})$, the resistivity of the CZTS samples exhibits an activated behavior obeying the nearest-neighbor hopping conduction mechanism. The activation energies, obtained for CZTS samples 1,2 and 3, were $49 \mathrm{meV}, 42 \mathrm{meV}$, and $43 \mathrm{meV}$, respectively. Using two different methods to study the conductivity of these samples at low temperature (below
$T=150 \mathrm{~K}$ ), we found that their resistivity behavior corresponds to the Mott variable-range hopping mechanism. The value of the energy of the acceptor level was calculated to be $W_{1} \approx 48 \mathrm{meV}$, $W_{1} \approx 42 \mathrm{meV}$, and $W_{1} \approx 41 \mathrm{meV}$ for samples CZTS 1,2 and 3, respectively. From the measurement of the Hall coefficient, the resulting semiconductor samples exhibit $p$-type conductivity. The carrier concentration of the CZTS samples was determined to be about $p \sim 10^{16} \mathrm{~cm}^{-3}$. Single-phase CZTS thin films were deposited using magnetron sputtering. Optical measurements were carried out using a spectrophotometer. The optical band gap of the CZTS thin film was found to be $1.3 \mathrm{eV}$. This makes these materials potentially important for photovoltaic applications.

\section{ACKNOWLEDGEMENTS}

This work was financially supported by the Ministry of Education and Science of the Russian Federation Reference Number 2014/420-367. The reported study was funded by RFBR according to the research Project No. 15-42-03192. This work was carried out using the scientific equipment of the joint research center "Diagnostics of structure and properties of nanomaterials" of the Belgorod National Research University, which is financially supported by Ministry of Science and Education of the RF under Project No. 14.594.21.0010, unique code RFMEFI59414X0010.

\section{REFERENCES}

1. S. Ahmed, K.B. Reuter, O. Gunawan, L. Guo, L.T. Romankiw, and H. Deligianni, Adv. Energy Mater. 20, 1 (2011).

2. M.I. Hoffert, K. Caldeira, A.K. Jain, E.F. Haites, L.D.D. Harvey, S.D. Potter, M.E. Schlesinger, S.H. Schneider, R.G. Watts, T.M.L. Wigley, and D.J. Wuebbles, Nature 395, 881 (1998).

3. Q. Guo, H.W. Hillhouse, and R. Agrawal, J. Am. Chem. Soc. 131,11672 (2009).

4. W. Ki and H.W. Hillhouse, Adv. Energy Mater. 1, 732 (2011).

5. T. Tanaka, T. Nagatomo, D. Kawasaki, M. Nishio, Q. Guo, A. Wakahara, A. Yoshida, and H. Ogawa, J. Phys. Chem. Sol. 66, 1978 (2005).

6. K. Tanaka, N. Moritake, and H. Uchiki, Sol. Energy Mater. Solar Cells 91, 1199 (2007).

7. M. Nakashima, J. Fujimoto, T. Yamaguchi, and M. Izaki, Appl. Phys. Express 8, 42303 (2015).

8. R. Nitsche, D.F. Sargent, and P. Wild, J. Cryst. Growth 1, 52 (1967).

9. W. Schafer and R. Nitsche, Mater. Res. Bull. 9, 645 (1974).

10. S. Chen, X.G. Gong, A. Walsh, and S.-H. Wei, Appl. Phys. Lett. 94, 041903 (2009).

11. C. Persson, J. Appl. Phys. 107, 053710 (2010).

12. J. Paier, R. Asahi, A. Nagoya, and G. Kresse, Phys. Rev. B 79, 115126 (2009).

13. A. Goetzberger, C. Hebling, and H. Schock, Mater. Sci. Eng. 40, 1 (2003).

14. T. Kato, H. Hiroi, N. Sakai, S. Muraoka, and H. Sugimoto, in 27th European Photovoltaic Solar Energy Conference (2012). doi:10.4229/27thEUPVSEC2012-3CO.4.2.

15. M. Jiang and X. Yan, Solar Cells-Research and Application Perspectives, ed. A. Morales-Acevedo (InTech, 2013). doi: $10.5772 / 50702$. 
16. H. Katagiri, K. Jimbo, S. Yamada, W.S. Maw, T. Fukano, T. Ito, and T. Motohiro, Appl. Phys. 1, 041201 (2008).

17. J. Rodríguez-Carvajal, Phys. B 192, 55 (1993).

18. P.A. Fernandes, P.M.P. Salomé, and A.F. da Cunha, J. Alloys Compd. 509, 7600 (2011).

19. M. Himmrich and H. Haeuseler, Spectrochim. Acta 47A, 933 (1991).

20. D. Yuanyuan, J. Wanqi, and L. Huanyong, J. Semicond. 30, 083005 (2009).

21. B.I. Shklovskii and A.L. Efros, Electronic Properties of Doped Semiconductors (Moscow: Science, 1979), p. 416.

22. D.M. Finlayson and P.J. Mason, J. Phys. C Solid State Phys. 19, 299 (1986).
23. K.G. Lisunov, M. Guk, A. Nateprov, S. Levcenko, V. Tezlevan, and E. Arushanov, Sol. Energy Mater. Sol. Cells 112, 127 (2013).

24. J.I. Pankove, Optical Processes in Semiconductors (Englewood Cliffs: Prentice-Hall, 1971), p. 422.

25. C.X. Wang, G.W. Yang, T.C. Zhang, H.W. Liu, Y.H. Han, J.F. Luo, C.X. Gao, and G.T. Zou, Diamond Relat. Mater. 12, 1548 (2003).

26. T. Shibuya, Y. Goto, Y. Kamihara, M. Matoba, K. Yasuoka, L.A. Burton, and A. Walsh, Appl. Phys. Lett. 104, 0219121 (2014). 